\title{
Quantum optimal control via gradient ascent in function space and the time-bandwidth quantum speed limit
}

\author{
Dennis Lucarelli* \\ Johns Hopkins University Applied Physics Laboratory \\ 11100 Johns Hopkins Road, Laurel, MD, 20723, USA
}

\begin{abstract}
A gradient ascent method for optimal quantum control synthesis is presented that employs a gradient derived with respect to the coefficients of a functional basis expansion of the control. Restricting the space of allowable controls to weighted sums of the Slepian sequences efficiently parameterizes the control in terms of bandwidth, resolution and pulse duration. A bound showing minimum time evolutions scaling with the inverse of the control bandwidth [S. Lloyd and S. Montangero, PRL, $113,010502,(2014)]$ is recovered and the method is shown numerically to achieve the bound on entangling two-qubit quantum gates.
\end{abstract}

\section{INTRODUCTION}

Function families play a significant role in quantum mechanics often appearing as eigenfunctions of the Schrödinger equation. Elementary examples include the Hermite polynomials that enter as factors of the wavefunctions of the quantum harmonic oscillator, the Legendre and Laguerre polynomials associated with the spectrum of the hydrogen atom, and more recently the Mathieu functions as eigenfunctions to the Cooper pair box Hamiltonian.

In the context of quantum information, functional analytic methods have been employed for quantum control, parameterizing the space of allowable controls and reducing the search space for optimal synthesis. Recent results include the numerical determination of dynamically corrected gates using Walsh functions [1, 2, the use of Hanning windows as a basis for frequency selective control of superconducting qubit devices [3] and the control protocol that uses randomized basis functions to parameterize the controls 4. These methods numerically locate optimal controls using a Nelder-Mead simplex method on the basis function coefficients. However, simplex based search methods generally scale poorly with the dimension of the unknown parameter [5], thus limiting the number of basis functions in the expansion of the control and the complexity of solutions amenable with this approach.

Gradient based optimization methods, while converging only to a local maximum, scale well with problem size dimension and are commonly used in high dimensional optimization problems. The GRAPE algorithm [6] introduced a gradient based approach for solving the optimal control problem for quantum gate synthesis. Using the well-known formula for parameter differentiation of an exponential operator, GRAPE calculates a gradient at each time-step of the controlled evolution and has been applied to a number of quantum control problems beyond its NMR origins. Calculating the gradient at each timestep independently, however, can lead to widely varying

\footnotetext{
* dennis.lucarelli@jhuapl.edu
}

control amplitudes at sequential times potentially violating limits on the slew rate or bandwidth of the control.

This paper introduces a method, Gradient Ascent in Function Space (GRAFS), that leverages standard gradient ascent solvers on the set of basis function coefficients parameterizing the entire time extent of the control. This functional analytic approach incurs little additional computational effort over GRAPE and offers a number of advantages, most notably, a significant dimensionality reduction of the underlying optimization problem and the ability to constrain the control manifold to smooth, well-behaved function families chosen to match the control problem at hand. The GRAFS method generalizes a gradient expression derived for control of spins in NMR [7] and subsequently used for optimal control of classical systems [8]. The gradient derived here is seen as a consequence of the product rule applied to the matrix product defined by a first-order Trotter expansion of the propagator, thus generalizing the functional gradient in [7] beyond two-level semi-classical systems.

Similarly, a recent method [9] constructs high-fidelity, analytic controls expressed in a basis function expansion. In that work, a dynamical equation is derived for the gradient of the propagator with respect to its parameters that couples to the Schrödinger dynamics. The adjoined system is then integrated to construct the gradient and used to maximize the objective function.

This paper also investigates the role of control bandwidth in quantum control problems. Constraints on the control bandwidth arise from practical considerations of experimental procedures and classical control electronics and are present in all modern qubit systems. With the focus on bandwidth, it is natural to consider the discrete prolate spheroidal sequences [10, commonly referred to as the Slepian sequences, as a basis for piece-wise constant controls. These finite length sequences faithfully and efficiently represent the space of band-limited signals and serve as the basis functions parameterizing the controls in this paper. Given the experimental constraints imposed on quantum controls, methods have been proposed to account for the effects of band-limited, bounded control in optimal control synthesis [11 14]. These methods introduce a penalty on the derivative of the control 
term in the control objective function, a Fourier cut-off constraint or a band-limiting filter in the control protocol iterates. The gradient method proposed here, with the aid of the Slepian sequences, constrains the space of controls a priori to produce an intrinsic control solution without additional terms in the objective function or post hoc band-limiting.

Closed quantum control systems have a convenient representation as control systems on Lie groups [15, [16] from which a number of properties can be immediately inferred. In particular, the Lie algebra rank condition (LARC), determined by the Lie algebra spanned by repeated commutators of the internal (or drift) Hamiltonian and the control Hamiltonians, can be used to determine the reachability of a quantum system. The reachability condition ensures the existence of controls that can reach any point in state space and is a generic condition for systems evolving on (compact) Lie groups [17, 18. The LARC, however, does not lead to a constructive procedure for determining the controls required to drive the system to a desired element of the Lie group. This control synthesis problem requires a solution to a twopoint boundary value problem and can solved analytically by pulse area theorems or in special cases (c.f. [19]). For more general, complex control situations, numerical methods from optimal control theory are often necessary.

Fundamental limits on the evolution time of a quantum system are often stated as quantum speed limits (QSL). These bounds are often geometric restatements of the time-energy uncertainty relation for time independent systems [20 22] and have been generalized to time-dependent, driven quantum systems [23 25]. Due to relatively short coherence times and slow logic gates, the QSL is an important limit in multiple qubit quantum gate synthesis 26]. Recently, the set of quantum states reachable in polynomial time with bounded controls has been characterized and an associated bound has been derived relating the control bandwidth, final state accuracy and minimum achievable evolution time 27. This time-bandwidth QSL has an intuitive appeal - higher bandwidth controls drive a quantum system to its target state at a faster rate. The gradient method introduced here approximately realizes this bound and thus demonstrates a control synthesis method that elucidates the limits of bounded, band-limited control.

The band-limited characteristics of Slepian-modulated controls as applied to qubits have recently been experimentally validated and deployed for narrowband spectroscopy of noise in a microwave control system [28. Further results and details on Slepian based noise spectroscopy can be found in the recent manuscript [29].

\section{THE SPACE OF TIME-BAND-LIMITED SEQUENCES}

The time-bandwidth uncertainty relation governs the concentration properties of classical signals. In the lim- iting case, the relation is observed by a pure tone with infinite time extent and vanishing spectral measure. As a purely mathematical object, the only signal that is both time and bandwidth limited is trivially zero. Motivated by problems in communications technology, where all signals are manifestly both time and band-limited, a theory of band-limited functions was developed by seeking a engineering compromise to the uncertainty relation 30 33. These functions are characterized by maximizing the fraction of a signals energy in a time interval while enforcing a band-limiting constraint. The solution to this optimization problem was aided by the discovery of a differential operator that simultaneously commutes with time and band-limiting operators. The form of this commuting operator is related to the wave equation in prolate spheroidal coordinates, thus christening the resulting functions as the prolate spheroidal wave functions (PSWF).

From a signals and control perspective, it is often useful to consider the discrete analogues to the PSWF, the discrete prolate spheroidal sequences or simply the Slepian sequences [10. For a sequence of length $N$ and half bandwidth $W$, the Slepian sequences, $v_{k}(N, W)$, are defined as real solutions to the eigenvalue problem

$$
\sum_{m=0}^{N-1} \frac{\sin 2 \pi W(n-m)}{\pi(n-m)} v_{k}(m ; N, W)=\lambda_{k}(N, W) v_{k}(\ell ; N, W)
$$

where $k, \ell \in\{0,1, \ldots, N-1\}$ and $v_{k}(\ell ; N, W)$ is the $\ell$-th element of $k$-th order Slepian. The eigenvalues $\lambda_{k}(N, W)$ can be shown to be a measure of spectral concentration and are ordered such that $1 \geq \lambda_{0}(N, W) \geq \lambda_{1}(N, W) \geq$ $\cdots \geq \lambda_{N}(N, W)$ and have the remarkable property that the first $2 N W$ eigenvalues are near unity, while the remaining eigenvalues are close to 0. In Ref. 34, Slepian uses this property to establish the approximate dimension of the space of band-limited sequences of length $N$ to be $2 N W$. The Slepian order, $k$, for the sequence $v_{k}(N, W)$, indicates the even/odd symmetry of the sequence with respect to its midpoint and its number of zero-crossings. In the following, weighted sums of Slepian sequences are identified as piecewise constant controls by specifying a sequence length $N$, a half bandwidth parameter $W \in(0,0.5)$ and a pulse duration $\tau$. The pair $(N, \tau)$ then defines a control resolution $\Delta t$, the associated Nyquist frequency and $W / \Delta t$ is in units of hertz.

\section{QUANTUM OPTIMAL CONTROL AND GRADIENT ASCENT IN FUNCTION SPACE}

For a closed, finite dimensional quantum system, the state dependence of the Schrödinger equation is often removed and the dynamics are equivalently lifted to a differential equation on the space of special unitary matrices $U \in S U(d)$, that act on the $d$-dimensional Hilbert space of quantum states. In this representation, the quantum 


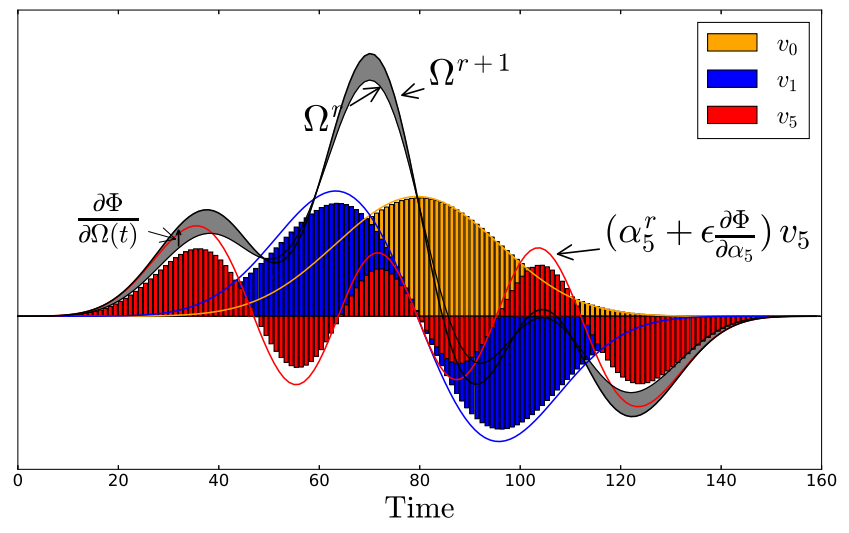

FIG. 1. (Color online) Depiction of GRAPE and GRAFS control iterates. GRAPE updates the control at each timestep $\left(\frac{\partial \Phi}{\partial \Omega(t)}\right)$, while GRAFS increments the basis function coefficient and affects the control at all times $\left(\frac{\partial \Phi}{\partial \alpha}\right)$. Slepian sequences, $v_{k}$, are shown for $k=0,1$, and 5

control system is given by

$$
\dot{U}(t)=-\frac{i}{\hbar}\left(H_{d}+\sum_{j=1}^{M} \Omega_{j}(t) H_{j}\right) U(t)
$$

where $H_{d}$ is the time-independent drift Hamiltonian and the $H_{j}$ are the control Hamiltonians that capture the coupling of the qubits to an externally applied control field. Denote the controlled Hamiltonian with drift as

$$
H_{\Omega}(t)=H_{d}+\sum_{j=1}^{M} \Omega_{j}(t) H_{j}
$$

The solution to (2) must account for the non-commuting operators $\left[H_{\Omega}\left(t_{\ell}\right), H_{\Omega}\left(t_{\ell}\right)\right] \neq 0$ for $k \neq \ell$ and is formally given by the time-ordered integral

$$
U_{\tau}(\Omega)=\mathbf{T} \exp \left[-\frac{i}{\hbar} \int_{0}^{\tau} H_{\Omega}(t) d t\right]
$$

With initial conditions given by the identity matrix, $U_{0}=$ $\mathbf{1}$, the solution of (2) simply propagates the state by matrix-vector multiplication $\psi_{\tau}=U_{\tau} \cdot \psi_{0}$.

The quantum gate synthesis problem is to determine the control functions $\left\{\Omega_{j}(t)\right\}_{j=1}^{M}$ that generate a desired target unitary $U_{\text {targ }}$ at some time $\tau$. Optimal control typically defines an objective function that is optimized subject to a dynamical constraint and initial and final conditions on the state of the system. In quantum gate synthesis, this methodology is employed to determine control fields that minimize trace distance from a target unitary while evolving according to the Schrödinger equation (2).

The optimal control objective function may take several forms depending on the control task, but all require a fidelity measure, $\mathcal{F}$, to be maximized. A common choice is the (global) phase invariant distance to the target unitary at the at the final time $\tau$

$$
\Phi(\mathcal{F}(\Omega))=\frac{1}{d}|\mathcal{F}(\Omega)|
$$

normalized by the dimension of the system, $d$, with the trace fidelity measure

$$
\mathcal{F}(\Omega)=\operatorname{tr}\left[U_{\text {targ }}^{\dagger} \cdot U_{\tau}(\Omega)\right]
$$

First order optimality conditions, known as Pontrayagin's maximum principle (PMP), are well established but, in general, result in a system of nonlinear differential equations that must be solved for optimal control synthesis. In Ref. [6], a Gradient Ascent Pulse Engineering algorithm (GRAPE) was introduced to numerically solve the optimal control problem that simplifies the optimization problem by iteratively updating the control fields by small steps in the gradient direction of the objective function at each time-step of the control. The pulse shaping iterates are of the form

$$
\Omega_{j}^{r+1}\left(t_{\ell}\right)=\Omega_{j}^{r}\left(t_{\ell}\right)+\epsilon \frac{\partial \Phi\left(\Omega^{r}\right)}{\partial \Omega_{j}\left(t_{\ell}\right)}
$$

with $r$ denoting the iteration number and the increment $\epsilon$ being chosen by line search. While consistent with the PMP, GRAPE derives its algorithmic simplicity by removing any explicit constraints on the control in the objective function. For a system without integral constraints on the controls, the PMP conditions result in trivial co-state dynamics, simplifying the first order necessary conditions [35] and ensuring that gradient ascent updates are sufficient for convergence to a local maximum.

Assuming a discretization of the controls into $N$ equal $\Delta t$ length segments with total pulse duration, $\tau=N \Delta t$, the GRAPE algorithm proceeds by using the product of exponentials

$$
U_{\tau} \approx e^{-\frac{i \Delta t}{\hbar} H_{\Omega}\left(t_{N}\right)} e^{-\frac{i \Delta t}{\hbar} H_{\Omega}\left(t_{N-1}\right)} \cdots e^{-\frac{i \Delta t}{\hbar} H_{\Omega}\left(t_{1}\right)}
$$

as a good approximation to the time-ordered integral (4) and the exact diagonalization formula [36] for parameter differentiation of the matrix exponential

$$
\frac{d}{d s} e^{A+s B}
$$

Let $\left\{\left|\lambda_{\nu}\right\rangle, \lambda_{\nu}\right\}$ denote an eigensystem for the matrix $A+$ $s B$, then the matrix elements of the partial derivative are given by

$$
\begin{aligned}
&\left\langle\lambda_{\nu}\right| \frac{\partial[A+s B]}{\partial s}\left|\lambda_{\mu}\right\rangle= \\
& \begin{cases}-i \Delta t\left\langle\lambda_{\nu}|B| \lambda_{\nu}\right\rangle e^{-i \Delta t \lambda_{\nu}} & \text { for } \nu=\mu \\
-i \Delta t\left\langle\lambda_{\nu}|B| \lambda_{\mu}\right\rangle \frac{e^{-i \Delta t \lambda_{\nu}-e^{-i \Delta t \lambda_{\mu}}}}{\lambda_{\nu}-\lambda_{\mu}} & \text { for } \nu \neq \mu\end{cases}
\end{aligned}
$$



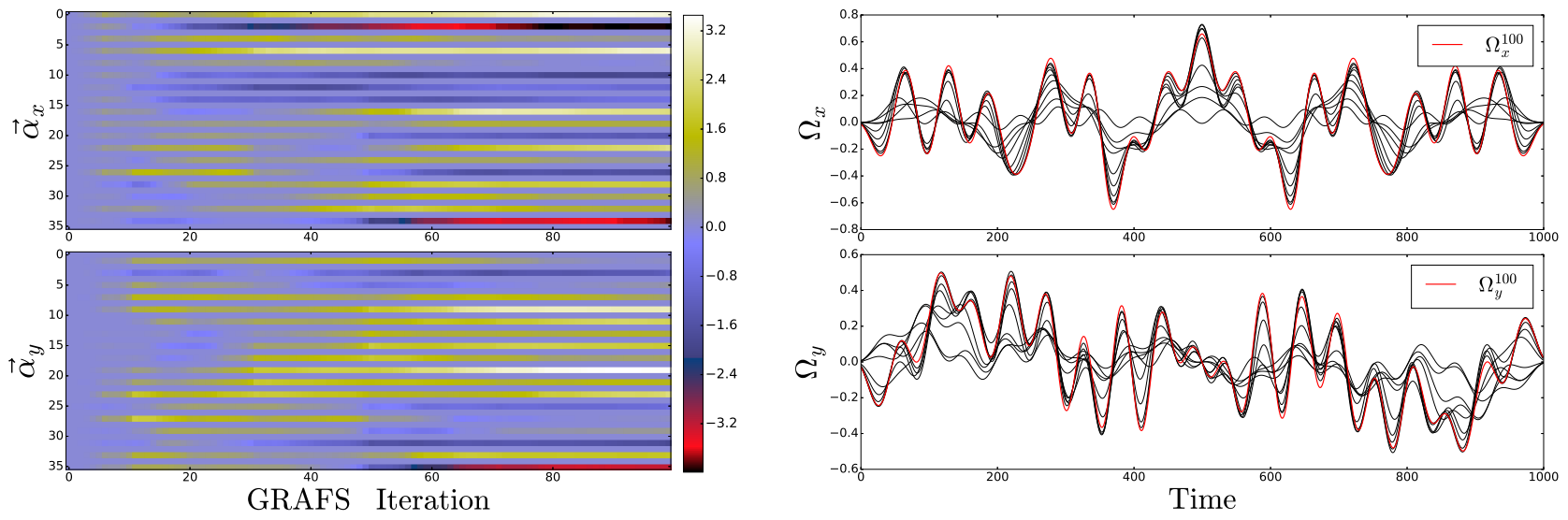

FIG. 2. (Color online) GRAFS iterations and convergence for constructing a Toffoli gate with two single qubit controls and Heisenberg exchange operations. The optimization trajectory of Slepian sequence weights is shown in the left panel and 10 pulse shaping iterations are shown on the right where $\Omega_{j}^{r}=\mathcal{V} \cdot{\overrightarrow{\alpha_{j}}}^{r}$. The final pulse shape is shown in bold red.

Define the notation

$$
U_{k: \ell}=U\left(t_{k}\right) \cdots U\left(t_{\ell}\right)
$$

for matrix products of the form (8). Taking the partial derivative of (8) with respect to the $j$-th control at time $t_{\ell}=\ell \Delta t$ yields

$$
\frac{\partial U_{\tau}}{\partial \Omega_{j}\left(t_{\ell}\right)}=U_{N: \ell+1} \frac{\partial U\left(t_{\ell}\right)}{\partial \Omega_{j}\left(t_{\ell}\right)} U_{\ell-1: 1}
$$

and by the chain rule we have

$$
\frac{\partial \Phi(\Omega)}{\partial \Omega\left(t_{\ell}\right)}=\frac{1}{d} \operatorname{Re}\left[e^{-i \arg (\mathcal{F})} \operatorname{tr}\left[U_{\text {targ }}^{\dagger} \cdot \frac{\partial U_{\tau}}{\partial \Omega_{j}\left(t_{\ell}\right)}\right]\right]
$$

where $\arg (\cdot)$ denotes the phase of a complex number. These expressions and their derivations can be found in 37. Denote the GRAPE gradient over all controls as the multi-dimensional tensor $\nabla_{\Omega} U_{\tau} \in \mathbb{C}^{N \times M \times d \times d}$ with components $\nabla_{\Omega_{j}}^{\ell} U_{\tau}$ given by 12 .

In contrast to updating the controls at each time-step, the GRAFS method first expresses the controls in a functional basis expansion and exploits the observation that the gradient of the propagator at the final time with respect to the the basis function coefficients is simply an application of the product rule on the matrix products defining the approximate propagator. The update rules are depicted in FIG. 1, with the essential difference being that variations of the basis function coefficients globally affect the control field over all times, whereas GRAPE updates affect each time-step independently.

The controls are formally expressed as weighted sums of length $N$ piecewise constant sequences $v_{k}(t)$

$$
\Omega_{j}\left(t_{\ell}\right)=\sum_{k=0}^{K} \alpha_{k j} v_{k}\left(t_{\ell}\right)
$$

with real coefficients $\alpha_{k j}$. While the method is applicable to any set of basis functions, the simulations in the following are performed with controls expressed as weighted sums of Slepian sequences. Applying the product rule to the matrix product $(8)$ and setting $\hbar=1$, the partial derivative is given by

$$
\frac{\partial U_{\tau}}{\partial \alpha_{k j}}=\sum_{\ell=1}^{N} U_{N: \ell+1} \frac{\partial U\left(t_{\ell}\right)}{\partial \alpha_{k j}} U_{\ell-1: 1}
$$

where

$$
\frac{\partial U\left(t_{\ell}\right)}{\partial \alpha_{k j}}=\frac{\partial \exp \left[-i \Delta t H_{\Omega}\left(t_{\ell}\right)-i \alpha_{k j} \Delta t v_{k}\left(t_{\ell}\right) H_{j}\right]}{\partial \alpha_{k j}}
$$

is of the form (9) with $A=-i \Delta t H_{\Omega}\left(t_{\ell}\right), B=$ $-i \Delta t v_{k}\left(t_{\ell}\right) H_{j}$ and $s=\alpha_{k j}$. Note that by the formula (10), the scalars $v_{k}\left(t_{\ell}\right)$ distribute out of the inner product to yield

$$
\frac{\partial U\left(t_{\ell}\right)}{\partial \alpha_{j k}}=v_{k}\left(t_{\ell}\right) \frac{\partial U\left(t_{\ell}\right)}{\partial \Omega_{j}\left(t_{\ell}\right)}
$$

Let $\mathcal{A}$ denote the matrix of coefficients so that $\mathcal{A}_{k j}=\alpha_{k j}$ and arrange the basis functions as columns of the $N \times K$ matrix $\mathcal{V}$. Using (17), the GRAFS gradient, $\nabla_{\mathcal{A}} U_{\tau} \in$ $\mathbb{C}^{K \times M \times d \times d}$, can be viewed as a contraction over the time index

$$
\nabla_{\mathcal{A}} U_{\tau}=\mathcal{V} \otimes_{\ell} \nabla_{\Omega} U_{\tau}
$$

with the matrix-valued components

$$
\left[\nabla_{\mathcal{A}} U_{\tau}\right]_{k j}=\sum_{\ell=1}^{N} \mathcal{V}_{\ell k} \nabla_{\Omega_{j}}^{\ell} U_{\tau}
$$

This tensor-dot operation can be efficiently implemented with the matrix of basis functions and the GRAPE gradient and thus the GRAFS gradient incurs little additional 


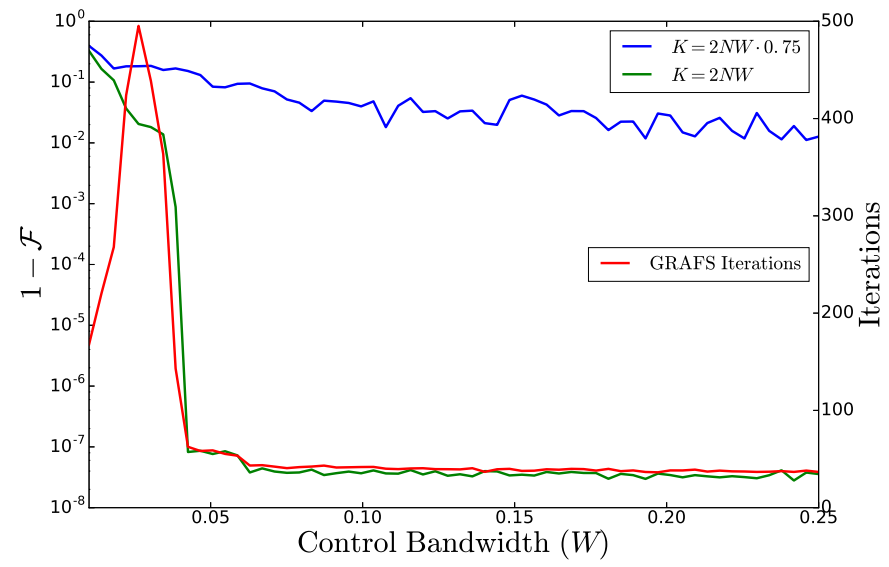

FIG. 3. (Color online) Infidelity as a function of bandwidth $(\mathrm{W})$ and number of Slepians sequences $(\mathrm{K})$. Also shown is the number of GRAFS iterations to convergence

computational overhead. Finally, the chain rule 13 can be applied to obtain $\nabla_{\mathcal{A}} \Phi$ and the gradient ascent control update in matrix form is simply

$$
\Omega^{r+1}=\mathcal{V} \cdot\left(\mathcal{A}^{r}+\epsilon \nabla_{\mathcal{A}^{r}} \Phi\right)
$$

An advantage of GRAFS is that control constraints are enforced a priori, through the use of basis functions, while still employing the gradient ascent procedure. Since arbitrary pulse shapes can often be faithfully represented by a small set of basis functions, GRAFS also constructs a more efficient optimization problem. In the case of Slepian parameterized controls, $2 N W$ sequences approximately characterize the space of band-limited sequences. For a system with $M$ controls, this results in an unknown parameter vector of dimension $2 N W M$ for GRAFS compared to $N M$ for GRAPE. Since $W$ is strictly (and typically much) less than 0.5, this represents a significant reduction in dimensionality of the underlying optimization problem.

An illustrative example of the method is given by the following notional quantum control system. Suppose the control task is to construct a three qubit Toffoli (or controlled-controlled-not) gate from Heisenberg exchange operators and two independent controls. Denoting the Heisenberg exchange on qubits $i$ and $j$ as $H_{\mathcal{X}}^{i j}$ and a single qubit control on qubit $k$ as $\sigma^{k}$ the system Hamiltonian is given by

$$
H_{\Omega}=H_{\mathcal{X}}^{12}+H_{\mathcal{X}}^{23}+\Omega_{x}(t) \sigma_{x}^{1}+\Omega_{y}(t) \sigma_{y}^{3}
$$

FIG. 2 shows the optimization trajectory of 100 iterations of the GRAFS algorithm using the L-BFGS algorithm to impose inequality constraints on the basis function weights $\left(\left|\alpha_{k j}\right|<5.0\right)$ and perform line search to calculate $\epsilon$ in the gradient update 20]. Controls were formed with a Slepian basis expansion with parameters $N=1000$ and $W=0.02$. Initial conditions for the coefficients for were set to zero. With these parameters, there are 40 Slepian sequences. Higher-order Slepians hold less

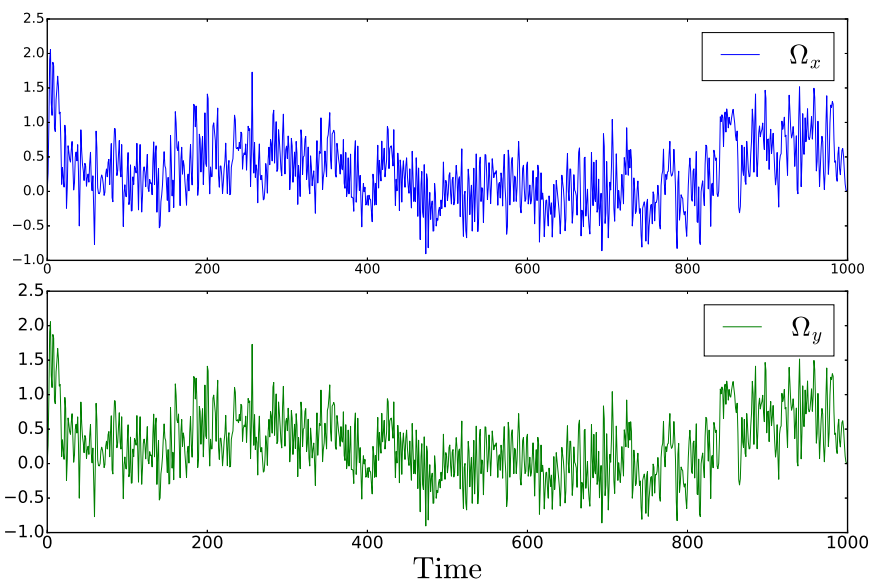

FIG. 4. (Color online) High bandwidth controls $(W=0.4)$ for system 21

spectral concentration and can have non-zero initial and final points. Removing these sequences leaves a final set of 36 sequences for pulse shaping. The final infidelity after 100 iterations was $\mathcal{O}\left(10^{-7}\right)$. All simulations were performed with the optimal control module in Qutip 38] by modifying the gradient calculation to (18) and the update equation to 20 .

Intuitively, controls constructed from high bandwidth Slepian sequences should contain more control authority and thus result in synthesis of high fidelity gates. FIG. 3 confirms this intuition and also shows the importance of using a nearly complete set of $K=2 N W$ Slepian sequences in the basis function expansion of the control. By varying the bandwidth and running the GRAFS procedure with the system 21, high fidelity Toffoli gates could not be generated across the entire bandwidth range ( $W \in[0.01, .25]$ shown)when using just three quarters of the available sequences. As shown in FIG. 3, convergence of the algorithm, as determined by the norm of the gradient getting small $\left(10^{-9}\right)$, also scales with the bandwidth of the control. High bandwidth controls, constructed from a Slepian basis with $N=1000, W=0.4$ and the GRAFS method, are shown in FIG. 4. These physically unrealizable controls approach the bang-bang limit and demonstrate how the Slepian sequences, with their continuous bandwidth parameter, represent the full space of controls.

\section{TIME-BANDWIDTH QUANTUM SPEED LIMIT}

Further analysis reveals a time-bandwidth relation by considering the QSL for time-dependent open quantum systems 23,25$]$

$$
\mathcal{T}_{Q S L} \geq \frac{\delta}{\frac{1}{\tau} \int_{0}^{\tau}\left\|H_{\Omega}(t)\right\|_{p} d t}
$$




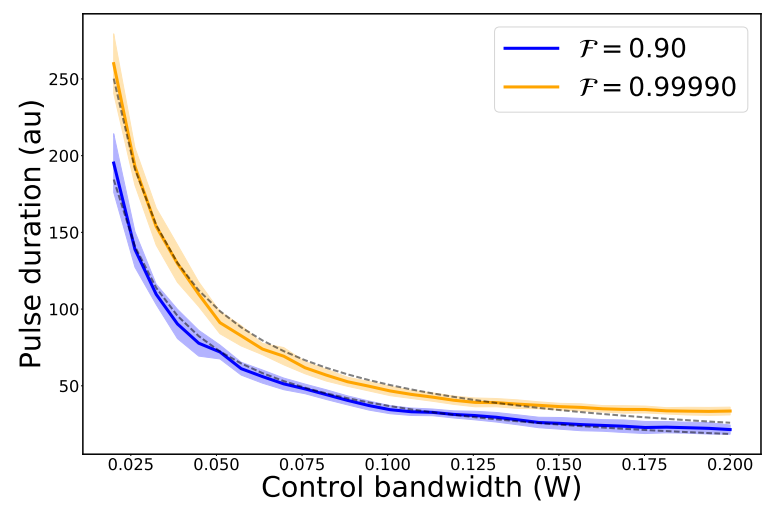

FIG. 5. (Color online) Empirical Quantum Speed Limit scaling for $\mathcal{F}=0.90$ (blue) and $\mathcal{F}=0.99990$ (orange). The fill regions denote the standard deviation across all 100 samples. Dashed lines are curve fits of the form $\frac{a}{W}+b$

where $\delta$ is an accuracy measure and $\|\cdot\|_{p}$ is the matrix $p$-norm. For mixed state transfer from $\rho_{0}$ to $\rho_{f}$, the accuracy measure is given in terms of the Bures angle $\delta=\sin ^{2}\left(\arccos \sqrt{\operatorname{tr}\left[\sqrt{\rho_{0}} \rho_{f} \sqrt{\rho_{0}}\right]}\right)$.

Restricting the space of allowable controls to the Slepian sequences and assuming closed, unitary dynamics and setting $p=2$, a bound on the denominator of 22 ) can be derived. Considering a single control Hamiltonian $H_{c}$, no drift term and denoting $\bar{H}_{\Omega}=\frac{1}{\tau} \int_{0}^{\tau}\left\|H_{\Omega}(t)\right\|_{2}$ and $\alpha=\max _{i}\left\{\alpha_{i}\right\}$ the following string of inequalities can be obtained

$$
\begin{aligned}
\bar{H}_{\Omega} & \leq \frac{1}{\tau} \sum_{i=1}^{2 N W} \int_{0}^{\tau}\left\|\alpha_{i} v_{i}(t) H_{c}\right\|_{2} d t \\
& =\frac{1}{\tau}\left\|\alpha H_{c}\right\|_{2} \sum_{i=1}^{2 N W} \sum_{\ell=1}^{N}\left|v_{i}\left(t_{\ell}\right)\right| \Delta t \\
& \leq \frac{1}{\tau}\left\|\alpha H_{c}\right\|_{2} \sum_{i=1}^{2 N W} \sqrt{N} \Delta t\left\|v_{i}\right\|_{2} \\
& =\frac{1}{\tau}\left\|\alpha H_{c}\right\|_{2} \sqrt{N} \Delta t 2 N W
\end{aligned}
$$

since $\left\|v_{i}\right\|_{1}<\sqrt{N}\left\|v_{i}\right\|_{2}$ and the Slepian sequences are normalized in two-norm. Setting $|\alpha|=\left\|H_{c}\right\|_{2}=1$ and since $\tau=N \Delta t$, a time-bandwidth QSL similar to Eq. 11 in Ref. [27]

$$
\mathcal{T}_{Q S L}(2 \sqrt{N} W) \geq \delta
$$

is easily recovered.

A series of numerical experiments were performed to qualitatively assess the $\mathcal{T}_{Q S L} \geq \mathcal{O}(\delta / W)$ scaling by running the GRAFS procedure repeatedly to determine the minimal time to achieve a specified fidelity given by the distance measure $\delta=\Phi(\mathcal{F})$. In these experiments, 100 target unitaries were constructed by random sampling parameters defining two-qubit gates in the Cartan decomposition. In particular, a tuple $\left(c_{x}, c_{y}, c_{z}\right)$ is sampled from the Weyl chamber [39] to form the target

$$
U_{\text {targ }}=K_{1} \exp \left(\frac{i}{2}\left(c_{x} \sigma_{x} \sigma_{x}+c_{y} \sigma_{y} \sigma_{y}+c_{z} \sigma_{z} \sigma_{z}\right)\right) K_{2}
$$

with randomly generated local operations $K_{i} \in S U(2) \otimes$ $S U(2)$. To further constrain the set of target gates, the tuples are formed by sampling from the Weyl chamber to obtain parameters from the 7 -faced polyhedron defining parameters for so-called perfect entanglers [39]. These are the two-qubit unitaries capable of generating maximally entangled states from an initial product state. This class of unitaries contains the well known quantum logic gates such as the CNOT and $\sqrt{\mathrm{SWAP}}$.

GRAFS was used to determine controls for this set of target gates while varying the control bandwidth, $W$, and keeping all other parameters, $\max _{i}\left\{\left|\alpha_{i}\right|\right\}=1$ and $N=1000$, fixed. The two-qubit Hamiltonian system with 4 independent controls is given by

$$
H_{\mathcal{X}}^{12}+\sum_{i=1}^{2} \Omega_{x}^{i}(t) \sigma_{x}^{i}+\Omega_{y}^{i}(t) \sigma_{y}^{i}
$$

A bracketing procedure, requiring multiple runs of the algorithm for each target gate, was used to estimate a minimal pulse duration for a given set of parameters and desired fidelity. Specifically, given a perfect entangler target, nominal control bandwidth, $W$, and pulse duration, $\tau$, the GRAFS algorithm is run until convergence or until reaching the desired fidelity. The algorithm maintains and updates three candidate times with differences forming a golden ratio. If the desired fidelity if obtained, the algorithm is run again with the same target but with a pulse duration decreased by the distance scaled by the golden ratio. If the algorithm converges without reaching the desired fidelity, the pulse duration is increased accordingly. Note that since the number of time steps, $N$, is fixed, changing the pulse duration effectively changes $\Delta t$ and the nominal bandwidth must be scaled as $W / \Delta t$, so that the effective control bandwidth is kept constant.

The results are shown in FIG. 5 with the mean of minimal times shown by the solid curve. The dashed lines are curve fits of the form $\frac{a}{W}+b$ showing approximate agreement with the mean. The filled region marks the standard deviation of minimal times across all samples. Some variance of minimal times is to be expected even when restricting the target set to the perfect entanglers. As shown in [40, the curvature of the SU(4) manifold distorts the volume elements (Haar measure) throughout the Weyl chamber, effectively making some target unitaries farther from the initial identity matrix. From FIG. 5 it is clear that the minimal time evolutions follow the time-bandwidth QSL scaling and demonstrate that Slepian based GRAFS not only recovers the bound of [27, but also gives a constructive procedure for realizing the bound. 


\section{CONCLUSION}

The Slepian sequences, with their continuously varying bandwidth parameter, efficiently represent the space of band-limited controls. A gradient expression on the coefficients of a basis function expansion of the control was derived and shown to accurately and efficiently determine optimal controls. Time optimal evolutions were numerically investigated and minimal pulse durations were shown to scale with the inverse of the control bandwidth. As quantum optimal control is increasingly applied to the experimental realm (cf. [14]), GRAFS may prove to be a useful technique for synthesizing band-limited timeoptimal controls. Specific applications to trapped ion and superconducting qubit systems are left for future work.

\section{ACKNOWLEDGEMENTS}

This project was supported by the Intelligence Advanced Research Projects Activity via Department of Interior National Business Center contract number 201212050800010. The author thanks Lorenza Viola and Michael J. Biercuk and Gregory Quiroz for helpful discussions.
[1] D. Hayes, K. Khodjasteh, L. Viola, and M. J. Biercuk, Phys. Rev. A 84, 062323 (2011).

[2] H. Ball and M. Biercuk, EPJ Quantum Technology 2 (2015).

[3] L. S. Theis, F. Motzoi, and F. K. Wilhelm, Phys. Rev. A 93, 012324 (2016).

[4] T. Caneva, T. Calarco, and S. Montangero, Phys. Rev. A 84, 022326 (2011)

[5] L. Han and M. Neumann, Optimization Methods and Software 21, 1 (2006).

[6] N. Khaneja, T. Reiss, C. Kehlet, T. Schulte-Herbrüggen, and S. J. Glaser, Journal of Magnetic Resonance 172, 296 (2005).

[7] T. E. Skinner and N. I. Gershenzon, Journal of Magnetic Resonance 204, 248 (2010).

[8] S. Meister, J. T. Stockburger, R. Schmidt, and J. Ankerhold, Journal of Physics A: Mathematical and Theoretical 47, $495002(2014)$.

[9] S. Machnes, E. Assmat, D. J. Tannor, and F. K. Wilhelm, "Gradient optimization of analytic controls: the route to high accuracy quantum optimal control," (2015), arXiv:1507.04261.

[10] D. Slepian, Bell System Technical Journal, The 57, 1371 (1978)

[11] F. Motzoi, J. M. Gambetta, S. T. Merkel, and F. K. Wilhelm, Phys. Rev. A 84, 022307 (2011)

[12] E. Pawela and Z. Puchała, Quantum Information Processing 13, 227 (2013)

[13] I. N. Hincks, C. E. Granade, T. W. Borneman, and D. G. Cory, Phys. Rev. Applied 4, 024012 (2015).

[14] R. W. Heeres, P. Reinhold, N. Ofek, L. Frunzio, L. Jiang, M. H. Devoret, and R. J. Schoelkopf, Nature Communications 8, 94 (2017).

[15] R. W. Brockett, SIAM Journal on Control 10, 265 (1972)

[16] V. Jurdjevic and H. J. Sussmann, Journal of Differential Equations 12, 313 (1972)

[17] W. M. Boothby, Journal of Differential Equations 17, 296 (1975)

[18] S. Lloyd, Phys. Rev. Lett. 75, 346 (1995).

[19] S. E. Economou and E. Barnes, Phys. Rev. B 91, 161405 (2015)

[20] I. T. L. Mandelshtam, Journal of Physics (USSR) 9, 249 (1945).

[21] Y. Aharonov and D. Bohm, Phys. Rev. 122, 1649 (1961)
[22] N. Margolus and L. B. Levitin, Physica D: Nonlinear Phenomena 120, 188 (1998).

[23] S. Deffner and E. Lutz, Phys. Rev. Lett. 111, 010402 (2013)

[24] M. M. Taddei, B. M. Escher, L. Davidovich, and R. L. de Matos Filho, Phys. Rev. Lett. 110, 050402 (2013).

[25] A. del Campo, I. L. Egusquiza, M. B. Plenio, and S. F. Huelga, Phys. Rev. Lett. 110, 050403 (2013).

[26] M. H. Goerz, T. Calarco, and C. P. Koch, Journal of Physics B: Atomic, Molecular and Optical Physics 44, 154011 (2011)

[27] S. Lloyd and S. Montangero, Phys. Rev. Lett. 113, $010502(2014)$

[28] V. M. Frey, S. Mavadia, L. M. Norris, W. de Ferranti, D. Lucarelli, L. Viola, and M. J. Biercuk, Nature Communications 8, 2189 (2017)

[29] L. M. Norris, D. Lucarelli, V. M. Frey, S. Mavadia, M. J. Biercuk, and L. Viola, "Optimally band-limited spectroscopy of control noise using a qubit sensor," (2018), arXiv: 1803.05538

[30] D. Slepian and H. Pollak, Bell System Technical Journal, The 40, 43 (1961).

[31] H. Landau and H. Pollak, Bell System Technical Journal, The 40, 65 (1961).

[32] H. Landau and H. Pollak, Bell System Technical Journal, The 41, 1295 (1962)

[33] D. Slepian, Bell System Technical Journal, The 43, 3009 (1964).

[34] D. Slepian, SIAM Review 25, 379 (1983).

[35] L. Pontryagin, V. G. Bolytanski, R. Gamkrekidze, and E. Mischenko, The Mathematical Theory of Optimal Procesess (The Macmillan Company, New York, NY, USA, 1964).

[36] K. Aizu, Journal of Mathematical Physics 4, 762 (1963)

[37] S. Machnes, U. Sander, S. J. Glaser, P. de Fouquières, A. Gruslys, S. Schirmer, and T. Schulte-Herbrüggen, Phys. Rev. A 84, 022305 (2011).

[38] J. Johansson, P. Nation, and F. Nori, Computer Physics Communications 183, 1760 (2012).

[39] J. Zhang, J. Vala, S. Sastry, and K. B. Whaley, Phys. Rev. A 67, 042313 (2003).

[40] J. V. Paul Watts, Maurice O'Connor, Entropy 15, 1963 (2015). 